\title{
COMMENTS
}

Comments are short papers which criticize or correct papers of other authors previously published in the Physical Review. Each Comment should state clearly to which paper it refers and must be accompanied by a brief abstract. The same publication schedule as for regular articles is followed, and page proofs are sent to authors.

\section{Hole projection, saddle points, and localization in the theory of autoionizing states}

\author{
Cleanthes A. Nicolaides \\ Theoretical and Physical Chemistry Institute, National Hellenic Research Foundation, 48 Vassileos Constantinou Avenue, \\ 11635 Athens, Greece \\ (Received 21 May 1991; revised manuscript received 30 July 1991)
}

\begin{abstract}
In view of a renewed interest in the theory of multiply excited resonances [M. Bylicki, Phys. Rev. A 39, 3316 (1989); 40, 1748 (1989); 41, 4093 (1990)] and the related comment by Chung [Phys. Rev. A 41, 4090 (1990)], I present a brief analysis of aspects of the theory for the identification and computation of resonances in many-electron systems. As examples, I use the $\mathrm{He}^{-} 2 s 2 p^{24} P$ and ${ }^{2} D$ triply excited resonances.

PACS number(s): $32.80 . \mathrm{Dz}, 34.80 .-\mathrm{i}$
\end{abstract}

\section{HOLE-PROJECTION IN TERMS OF ONE-PARTICLE ORTHOGONALITY CONSTRAINTS}

The papers [1-9] that present and discuss the "saddlepoint technique" for the computation of atomic resonances are characterized by three concepts, which justify and qualify the computations. The first is the application of a hole-projection operator to a trial wave function so as to achieve convergence of the variational calculation to the proper root. The second is the variation of a single parameter of the hole orbital (hydrogenic function) so as to maximize the total energy and then identify the saddle point as the unshifted resonance position. The third is the use of the solution thus obtained on the real axis in combination with a new term representing the asymptotic part of the resonance with complex coordinates so as to obtain total widths [8].

The idea and first practical implementation of shellstructure-dependent orthogonalities of trial functions of arbitrary $N$-electron autoionizing states in variational calculations were presented by the author [10,11], together with other aspects of the theory of resonances including the justification of doing state-specific Hartree-Fock (HF) or multiconfiguration Hartree-Fock (MCHF) calculations based on the concept of localization and its relation to the shell structure, number of nodes, square integrability of solutions, and the virial theorem. Use of one-electron projection operators in terms of Hartree-Fock orbitals implies the exclusion of undesirable $N$-electron lower states and open channels while providing upper bounds to third order in the total energy [12].

In Ref. [10] the orthogonality operators for the $N$ electron problem were written as $q_{i}=1-|n l\rangle\langle n l|$, where $|n l\rangle$ is the core orbital on which orthogonality of the orbital or correlated trial functions is imposed. (See p. 2088 of Ref. [10].) Consider a three-electron correlated trial wave function representing the square-integrable part $\Psi_{0}$ of an autoionizing state, which we wish to optimize variationally subject to orthogonality constraints to the hydrogenic $1 s$ orbital. If for simplicity's sake we choose the Schmidt orthogonalization to effect only one prespecified spin orbital, then it follows from Ref. [10] that ( $A$ is the antisymmetrizer)

$$
\begin{aligned}
\Psi_{0}^{\mathrm{tr}} & =A q_{1 s} \Psi_{0}\left(r_{1}, r_{2}, r_{3}\right) \\
& =A(1-|1 s\rangle\langle 1 s|) \Psi_{0}\left(r_{1}, r_{2}, r_{3}\right),
\end{aligned}
$$

which is exactly the form used by Chung in the "holeprojection method" [1-9].

As an example, consider the case of the $\mathrm{He}^{-}$ $1 s 2 s 2 p^{2} P^{\circ}$ resonance discussed by Chung [1] and Bylicki [3]. Chung initially studied this state in Ref. [5]. On p. 1080 he states that for a $|1 s 2 s 2 p\rangle$ function, the $1 s$ orthogonality would apply to the $2 s$ orbital. The resulting expression from Eq. (1) [his Eq. (5)] would be a function of the form

$$
|1 s 2 s 2 p\rangle+c\left|1 s 1 s^{\prime} 2 p\right\rangle
$$

where the $1 s^{\prime}$ spin orbital is different from the $1 s$ (see below). It is clear that this wave-function form emerges directly if the $2 s$ orbital of a $1 s 2 s 2 p$ configuration is Schmidt orthogonalized to a $1 s^{\prime}$ function. Alternatively, as it can be seen from the theory of Komninos, Aspromallis, and Nicolaides [13] of the $\mathrm{He}^{-} 1 s 2 s 2 p^{2} \mathrm{P}^{\circ}$ resonance, this form, albeit with $1 s=1 s^{\prime}$, can be obtained directly from a state-specific multiconfigurational Hartree-Fock calculation as

$$
\phi=0.995|1 s 2 s 2 p\rangle-0.099\left|1 s^{2} 2 p\right\rangle
$$

[Eq. (10) of Ref. [13]].

In the theory of Ref. [10], the hole projector is built in terms of state-specific Hartree-Fock orbitals correspond- 
ing to the core of lower states. On the other hand, Chung chooses the $q_{i}$ as a very simple function that is optimized in the search for a saddle point of the eigenvalue. Let us see the background and utility of this approach.

\section{CHOICE OF ORBITAL PROJECTORS AND THE MINIMUM-MAXIMUM THEOREM: THE OPTIMAL CHOICE FOR LOCATING THE EXACT POSITION OF A RESONANCE}

Higher roots of Hamiltonian matrices of interacting levels show saddle points [14]. This property was used by Chung [4] in connection with $q_{i}$ to offer a "proof of a theorem" (see the abstract and Sec. IV of Ref. [4]) for the general many-electron problem of resonances. I agree with Bylicki who pointed out $[2,3]$ the serious weaknesses of this "proof." Furthermore, I point out the fact that the minimum-maximum (minimax) property of eigenvalues [14] had already been studied in connection with the theory of resonance states. Perkins [15] identified this property as a possible practical tool for understanding the stabilization of roots expected to represent resonances. I quote excerpts from his abstract: "We vary constraint parameters so as to maximize the minimum energy which is accessible subject to constraints. By maximum-minimum property of eigenvalues, this is equivalent to taking suitable root of unconstrained secular equation... ." "This explains 'stabilization of roots' in Holoien's calculations, but the method will be most useful for atoms with more than two electrons."

Later, Dalgarno and Drake [16], in an article entitled "An energy maximization method for autoionizing states" proposed the saddle point of the lowest eigenvalue, determined with respect to one-electron radial nodal properties, as a computational element for the recognition of resonant states. They demonstrated their idea on the $\mathrm{He}$ $2 s 2 p^{3,1} P^{\circ}$ states. Finally, Hahn [17] also discussed the minimax theorem in relation to the "extremal properties of resonance eigenvalues."

As a heuristic tool, the minimax property of eigenvalues is indeed useful in the context of the proposals of Perkins [15], of Dalgarno and Drake [16], and of Hahn [17]. However, achieving a saddle point of a particular root as a function of one parameter in the hole orbital does not imply more rigor or a better accuracy than the case where the hole orbital is fixed from the Hartree-Fock shell structure $[10,11,18]$. The exact position of the resonance is determined only when the effects of the open channels are taken into account and this causes a shift of the initial energy whose exact magnitude and sign depend on the function spaces used. In order to underline this point, I consider the following. Bylicki has recently reported [19] results on the $\mathrm{He}^{-} 2 s 2 p^{24} P$ resonance. $\mathrm{He}$ compares two variational calculations, both carried out with the orthogonality constraint achieved by the application of system-dependent one-electron projection operators [10]. In the first type, the $1 s$ orbital is fixed as hydrogenic $\mathrm{He}^{+}$. In the second type, the structure concept is again used, only that now the orbital is optimized by maximizing the eigenvalue as a function of a nonlinear parameter in the $1 s$ orbital. Bylicki obtained an energy of
$57.420 \mathrm{eV}$ with the optimized hole projector and 57.395 $\mathrm{eV}$ with the unoptimized one. These results suggest that, once orthogonality to the physically important orbital has been imposed [10], the approximation of optimizing the one-parameter hole orbital according to the minimax theorem does not add any physically significant quantitative information. In other words, the association of the $\mathrm{He}^{-} 2 s 2 p^{24} P$ resonance with the energy $57.420 \mathrm{eV}$ rather than with $57.395 \mathrm{eV}$ is an arbitrary choice. (The difference of $0.025 \mathrm{eV}$ is the same as the energy shift $\Delta$, which was computed [20] to be $0.027 \mathrm{eV}$.)

An important result would indeed be the one stating that, given a trial square-integrable $\Psi_{0}$ representing a many-electron resonance, at the saddle of the corresponding eigenvalue of a variational calculation the energy shift due to the interaction with the continuum is minimized. However, such a proof has not yet been produced.

On the other hand, if the failure to obtain a saddle in a particular implementation of the minimax theorem implied beyond any doubt the nonexistence of an autoionizing state, as in Chung's conclusion [7,9] of the absence of the $\mathrm{He}^{-2} D$ resonance, then the criterion of hole-orbital optimization in the determination of resonance energies and wave functions would indeed be necessary. However, the new results [20] on $\mathrm{He}^{-} 2 s 2 p^{22} D$, verify the existence of this resonant state, suggesting that Chung's interpretation of his results is unfounded.

\section{CASE OF THE $\mathrm{He}^{-2 s} 2 p^{22} D$ RESONANCE AND THE STATE-SPECIFIC THEORY OF RESONANCES}

The story of the $2 s 2 p^{22} D$ resonance as seen through the Chung writings $[7,9]$ is as follows. In the 1960 s and early 1970s, experimentalists $[21,22]$ and theoreticians [10,23-27] alike agreed on the existence of the $\mathrm{He}^{-}$ $2 s 2 p^{22} D$ resonance around $58.3 \mathrm{eV}$ above the $\mathrm{He}$ ground state. Chung [7] and Chung and Davis [9] disputed the correctness of this work and claimed that this resonance does not exist. The arguments were based on their unconverged, saddle-point-technique calculations and on a critical appraisal of the previous theoretical work. For example, in Ref. [9], p. 94, they conclude, "This result does not corroborate the previous assignment by Fano and Cooper. What has been seen in the experiment could be the result of a postcollision interaction effect."

On discussing the particularities of this state, Chung [7] stressed that "one must exclude the $\left[(s, s)^{1} S d\right]$ and $\left[(p, p){ }^{1} S d\right]$ angular terms in the variational calculation," otherwise it will either collapse to the He $2 s^{2} S$ energy or it will not be very meaningful. Then he pointed to the characteristics of the Nicolaides wave function [10], as quoted by Schulz [22], p. 406, which contained $83 \%$ of HF $2 s 2 p^{2}$ and $17 \%$ from the correlation vectors, with the main contribution coming from $2 s^{2} n d$ and $2 p^{2} n d$ configurations. Thus he concluded that these early calculations did not offer "conclusive evidence for the existence of this Feshbach resonance since the inclusion of these terms may lead to erroneous results."

The answer to these remarks is based on the very essence of the nature of the state-specific theory (SST) of autoionizing states $[10,11,18,28-31]$. By the end of the 
1960s, the many-electron problem for nonstationary states embedded in the continuous spectrum presented conceptual and computational problems beyond those characterizing the ordinary ground state. The SST for such excited states has been formulated so as to be able to treat the many-electron problem for nonstationary states in the continuous spectrum, regardless of the number of electrons and of the features of the multichannel continuum. Furthermore, as regards the systematic computation, the foundations of the theory are practical. This feature is essential. For although when dealing with model potentials supporting nonstationary states it is possible to carry out experimental computations (e.g., vary parameters of interaction strength or of box size) in order to see some of the wave-function characteristics, for real polyelectronic atoms and molecules this luxury is prohibitive.

Briefly, the SST puts emphasis on the dynamical localization that must characterize all $N$-electron autoionizing states regardless of their mode of excitation or decay, and on the recognition that this can best be brought out from an MCHF (or similar) calculation of the important zeroth-order near-degeneracy effects. Since MCHF theory is variational, the justification for its implementation to the calculation of states embedded in the continuum was the shell-structure constraint of orthogonality to core orbitals, the proper orbital nodal structure, and the satisfaction of the virial theorem, a property which is intrinsic to converged $\mathrm{HF}$ or MCHF solutions as well as to localized systems. Thus the theory associates MCHF zeroth-order solutions with physical, quasilocalized states in the continuous spectrum.

A converged MCHF solution means that at the particular energy of the continuous spectrum this solution has the largest coefficient in the full expansion of the wave function. The correlating orbitals describe state-specific localized correlations and not open channels. For example, in the case of the $2 s 2 p^{22} D$ state the MCHF $3 d$ bound orbital of the $2 s^{2} 3 d$ correlating configuration is square integrable and nodeless and does not resemble the $\varepsilon d$ scattering functions. Yet, since this $3 d$ function is obtained independently of the $\varepsilon d$ channel and is not made orthogonal to it, it contains contributions from the continuous spectrum, just as the correlating bound orbitals of ground states do. The net overall effect is that the $2 s^{2} 3 d$ eigenvalue is above the $2 s 2 p^{2}$ solution, which corresponds to the lowest root. Thus, in the state-specific variational scheme, whether using an analytic HF $2 s 2 p^{2}$ solution and radially compact virtual orbitals of $d$ symmetry [10] or the numerical MCHF solution as in this work, the optimized $2 s^{2} 3 d$ and $2 p^{2} 3 d$ configurations simply contribute to the localized $\Psi_{0}$.

This can be further understood from the arguments of Ref. [11] (pp. 460-465) and of Ref. [18(b)], p. 109. That is, the correlating virtual orbitals, such as the $3 d$ in the $\mathrm{He}^{-}$case, pick up just a part of the continuous spectrum, which does not destroy the square integrability of the zeroth-order function. Of course, the remaining physically important portion of the continuum must be computed via an appropriate theory [18,28-31].

In order to demonstrate the above, and hence the ex- istence of the ${ }^{2} D$ resonance, a numerical MCHF calculation with all the $n=2$ and 3 configurations was carried out [20] while keeping the MCHF $2 s$ and $3 s$ orbitals orthogonal to the $1 s$. The converged solution has a weight of $86 \%$ for the $2 s 2 p^{2}$ configuration, in agreement with my previous calculation [10], which gave $83 \%$.

\section{EXTENSION TO THE COMPLEX ENERGY PLANE}

The third item is the "saddle-point complex-rotation method" [8]. The essence of this method is that once you have obtained the real energy $E_{0}$ and wave function $\Psi_{0}$ representing the square-integrable part of the resonance, then by adding terms with complex coordinates representing the asymptotic part of the resonance, a practical approach to the computation of widths is achieved. This is exactly the theory presented earlier in Refs. $[11,28-31]$ and since applied to inner-hole states such as $\mathrm{Ne}^{+} 1 s 2 s^{2} 2 p^{62} S$ [30] as well as to doubly excited states such as $\mathrm{He} 2 s 2 p{ }^{1} P^{\circ}[28]$ and $\mathrm{H}^{-} n s^{2}{ }^{1} S, n=3-7$ [29].

Our theory emphasized the relation between asymptotic boundary conditions and eigenvalues of the full or of effective Hamiltonians, the significance of separating the one- or the $N$-particle function spaces according to the physics of decay, and the concomitant simplification of the many-body problem based on matrix-element invariances upon rotation. In particular, the state-specific, square-integrable resonance function is written, in the context of different discussions, as

$$
\widetilde{\Psi}=\Psi_{0}+\sum_{n} a_{n} \widetilde{u}_{n},
$$

where $u_{n}$ is a set of complex functions for the asymptotic region (Eq. 7.7 of Ref. [11]) or, as

$$
\Psi(\rho)=a(\theta) \Psi_{0}(\rho)+b(\theta) X_{\mathrm{as}}(\rho), \quad \rho=r e^{i \theta}
$$

with

$$
\left\langle\Psi_{0}(\rho)|H(\rho)| \Psi_{0}(\rho)\right\rangle=\left\langle\Psi_{0}|H| \Psi_{0}\right\rangle=E_{0},
$$

and related simplifications due to the back rotation for the $N$-particle off-diagonal matrix elements, and

$$
\langle\Psi|H| \Psi\rangle=E_{0}+\Delta-\frac{i}{2} \Gamma .
$$

$\Psi_{0}$ is the square-integrable projection on the real axis containing the localized components [see Eqs. (1)-(4) of Ref. [10]] and $X_{\text {as }}$ (or, the basis set $\widetilde{u}_{n}$ ) represents the asymptotic component carrying the information on the energy shift $\Delta$ and width $\Gamma$.

In their "new procedure" (see abstract of Ref. [8]), Chung and Davis also employ a fixed $E_{0}$ from a calculation of $\Psi_{0}$ on the real axis and obtain $\Delta$ and $\Gamma$ upon the addition of new functions, [see my Eqs. (2) and (3)], which yield this information via the diagonalization of the nonHermitian Hamiltonian matrix. In their conclusion (Ref. [8], p. 3282), Chung and Davis state how "their" method differs from that of Junker and Huang. On the other hand, they fail to mention the fact that they have simply carried out an application of the theory of Refs. $[11,28,31]$.

The reader can easily make comparisons of the published work. However, I think that it is immediately helpful if I quote from the 1978 Nicolaides and Beck pa- 
per [11] and from the 1982 Chung and Davis paper [8]. We stated (Ref. [11], p. 506) "I.e., an arbitrarily chosen set of square-integrable functions may be a good representation of the real axis, but in the $\theta$ plane they need not." Chung and Davis in justifying "their" approach, state (Ref. [8], p. 3279), "The nonlinear parameters in $\Phi_{j}$ which are most suitable for the solution of $H\left(R_{N}, \Omega_{N}\right)$ may not be suitable for that of $H\left(R_{N} e^{i \theta}, \Omega_{N}\right)$."

An application [20] of our approach to the computation of the width of the aforementioned $\mathrm{He}^{-} 2 s 2 p^{24} \mathrm{P}$ resonance through the diagonalization of a small, $11 \times 11$ complex matrix, yields $\Delta=0.027 \mathrm{eV}$ and $\Gamma=0.015 \mathrm{eV}$.

\section{CONCLUSION}

In view of the recent publications $[1,3]$ on aspects of the calculation of the square-integrable part of resonances and the statement [1] as to the origin of the "saddle-point technique," I have brought to the reader's attention the fact that all the important elements of this approach, one-electron-core orthogonalities, identification of resonances with ieigenvalue saddles, two-step computation of complex eigenvalues via use of complex scaling etc., were in the literature $[10,11,15-$ $18,28-31$ ] long before 1979 [4] or 1982 [8].

Furthermore, by reviewing and emphasizing aspects of the state-specific approach to resonances, I have argued that the $\mathrm{He}^{-} 2 s 2 p^{22} \mathrm{D}$ resonance is real, regardless of whether it is above or below the He $2 s 2 p^{3} P^{\circ}$ threshold [20]. The fundamental reason for the reality of this resonance is the fact that well-converged self-consistent multiconfigurational calculations establish its localization. Such a wave function contains the important longrange correlations and dynamical screening effects while satisfying the virial theorem and the correct zeroth-order nodal structure and boundary conditions.

For many-electron systems, the one-electron-core orthogonality constraints [10] may be implemented within an electronic-structure-dependent theory $[10,11,13,18]$ or by optimizing a parametrized oneelectron function while searching for the saddle of the eigenvalue [15,16,1-9]. Perkins's proposal [15] to associate the variationally obtained saddles of certain eigenvalues of the Hamiltonian matrix with resonance positions, is an important heuristic tool for computational methods that depend on configuration-interaction expansions with arbitrary square-integrable basis sets [16,1-9]. However, since there is no way of predicting quantitatively the effect of the open channels, given a very accurate squareintegrable representation of a resonance, it is still unknown whether the exact resonance position is closer to the saddle or not.
[1] K. T. Chung, Phys. Rev. A 41, 4090 (1990).

[2] M. Bylicki, Phys. Rev. A 39, 3316 (1989); 40, 1748 (1989).

[3] M. Bylicki, Phys. Rev. A 41, 4093 (1990).

[4] K. T. Chung, Phys. Rev. A 20, 1743 (1979).

[5] K. T. Chung, Phys. Rev. A 23, 1079 (1981).

[6] K. T. Chung and B. F. Davis, Phys. Rev. A 22, 835 (1980).

[7] K. T. Chung, Phys. Rev. A 22, 1341 (1980).

[8] K. T. Chung and B. F. Davis, Phys. Rev. A 26, 3278 (1982); B. F. Davis and K. T. Chung, ibid. 29, 1878 (1984).

[9] K. T. Chung and B. F. Davis, in Autoionization, edited by A. Temkin (Plenum, New York, 1985), p. 73.

[10] C. A. Nicolaides, Phys. Rev. A 6, 2078 (1972). This reference cites the various approaches which had been applied to resonant states until 1971. At that time, the variational calculations were restricted by the Feshbach projection $P$ and $Q$ formalism to only two-electron systems, since they depended on the knowledge of the exact target states (Feshbach's asymptotic condition for the open channels). The two-electron bottleneck was bypassed by the formalism and computational approach of this work.

[11] C. A. Nicolaides and D. R. Beck, Int. J. Quantum Chem. 14, 457 (1978).

[12] In Ref. [10], pseudocontinuum functions representing open-channel "semi-internal" correlation effects were included separately via the diagonalization of small matrices, where care was exercised to avoid near degeneracies with the HF zeroth-order function.

[13] Y. Komninos, G. Aspromallis, and C. A. Nicolaides, Phys. Rev. A 27, 1865 (1983).

[14] For example, see J. W. Dettman Mathematical Methods in Physics and Engineering, 2nd ed. (McGraw-Hill, New York, 1969), Chap. 3.
[15] J. F. Perkins, Bull. Am. Phys. Soc. 13, 80 (1968); Phys. Rev. 178, 89 (1969).

[16] A. Dalgarno and G. W. F. Drake, Chem. Phys. Lett. 11, 509 (1971).

[17] Y. Hahn, Phys. Rev. A 5, 1607 (1972).

[18] (a) C. A. Nicolaides, Y. Komninos, and D. R. Beck, Phys. Rev. A 27, 3044 (1983); (b) Y. Komninos, N. Makri, and C. A. Nicolaides, Z. Phys. D 2, 105 (1986).

[19] M. Bylicki, Phys. Rev. A 45, 2079 (1992).

[20] C. A. Nicolaides (unpublished); Th. Mercouris, Y. Komninos, G. Aspromallis, and C. A. Nicolaides (unpublished).

[21] C. E. Kuyatt, J. A. Simson, and S. R. Mielczarek, Phys. Rev. 138, A385 (1965); J. J. Quémener, C. Paquet, and P. Marmet, Phys. Rev. A 4, 494 (1971); P. J. Hicks, C. Cvejanovic, J. Comer, F. H. Read, and J. M. Sharp, Vacuum 24, 573 (1974).

[22] G. J. Schulz, Rev. Mod. Phys. 45, 378 (1973).

[23] U. Fano and J. W. Cooper, Phys. Rev. 138, A400 (1965).

[24] I. Eliezer and Y. K. Pan, Theor. Chim. Acta (Berlin) 16, 63 (1970).

[25] K. Smith, D.E. Golden, S. Ormonde, B. W. Torres, and A. R. Davis, Phys. Rev. A 8, 3001 (1973).

[26] M. Ahmed and L. Lipsky, Phys. Rev. A 12, 1176 (1975).

[27] R. K. Nesbet, Phys. Rev. A 14, 1326 (1976).

[28] C. A. Nicolaides, Y. Komninos, and Th. Mercouris, Int. J. Quantum Chem. Symp. 15, 355 (1981).

[29] M. Chrysos, Y. Komninos, Th. Mercouris, and C. A. Nicolaides, Phys. Rev. A 42, 2634 (1990).

[30] C. A. Nicolaides and Th. Mercouris, Phys. Rev. A 32, 3247 (1985).

[31] C. A. Nicolaides and D. R. Beck, Phys. Lett. A 65, 11 (1978). 